\title{
Revisiter la Défense des femmes (1726) de Benito Jerónimo Feijoo
}

Revisiting Benito Jerónimo Feijoo's “Defence of women” (1726)

\section{Mónica Bolufer Peruga}

Traducteur : Rudy Chaulet

\section{OpenEdition Journals}

\section{Édition électronique}

URL : http://journals.openedition.org/clio/13005

DOI : 10.4000/clio. 13005

ISSN : $1777-5299$

\section{Éditeur}

Belin

Édition imprimée

Date de publication : 1 juin 2016

Pagination : 223-249

ISBN : 978-2-7011-9852-1

ISSN : $1252-7017$

\section{Référence électronique}

Mónica Bolufer Peruga, « Revisiter la Défense des femmes (1726) de Benito Jerónimo Feijoo », Clio. Femmes, Genre, Histoire [En ligne], 43 | 2016, mis en ligne le 01 juin 2019, consulté le 02 janvier 2020. URL : http://journals.openedition.org/clio/13005 ; DOI : 10.4000/clio.13005 


\title{
Varia
}

\section{Revisiter la Défense des femmes (1726) de Benito Jerónimo Feijoo ${ }^{1}$}

\author{
Mónica BOLUfer PERUGA
}

C'est une belle gageure que je tiens là. Ce n'est plus seulement le vulgaire ignorant avec qui j'entre en querelle : défendre toutes les femmes, cela revient à offenser presque tous les hommes ; car rare est celui qui ne s'attache pas à la préséance de son propre sexe par le mépris de l'autre ${ }^{2}$.

La Défense des femmes, publiée pour la première fois en 1726 par le religieux et homme de lettres espagnol Benito Feijoo, est l'un des textes les plus connus de la «querelle des femmes» du XVIII" siècle espagnol. Cet essai, très discuté dès sa publication, fut à l'origine

1 Ce travail fait partie du projet de recherche HAR2014-53802-P financé par le ministère espagnol de l'Économie et de la Compétitivité. Une première version, différente, a été présentée au colloque L'engagement des hommes pour l'égalité des sexes (Paris, 2010). Je remercie Florence Rochefort de l'occasion qui m'a été offerte de pouvoir présenter ce texte à ce colloque, ainsi que Juan Gomis Coloma pour les références fournies sur les tomes IV et VIII du Teatro crítico.

2 Feijoo 1778-1779 : 325. «En grave empeño me pongo. No es ya sólo un vulgo ignorante con quien entro en la contienda : defender a todas las mujeres, viene a ser lo mismo que ofender a casi todos los hombres : pues raro hay que no se interese en la precedencia de su sexo con desestimación del otro ». 
d'une longue et intense polémique qui est considérée comme un tournant dans l'histoire de ce débat. Sur ce point comme sur d'autres, l'historiographie classique a considéré Feijoo comme un précurseur isolé, une figure solitaire dans le supposé désert intellectuel de l'Espagne de la première moitié du XVIII siècle $^{3}$. Depuis un certain nombre d'années, cette interprétation qui met en exergue la rupture opérée par Feijoo a été révisée, et un autre récit a été proposé, qui fait remonter l'origine des Lumières espagnoles aux dernières décennies du XVII ${ }^{e}$ siècle. Cependant, il reste encore à approfondir ses apports au débat sur la différence des sexes, et à les replacer plus précisément dans leur contexte. Une analyse approfondie de son œuvre, vaste et diverse, prêtant une attention particulière à ses sources mais aussi à ses éventuelles contradictions et à ses paradoxes, devrait permettre de complexifier le récit de l'évolution du discours sur la différence des sexes au Siècle des Lumières.

Cet article prétend contribuer à cette relecture. Celle-ci semble particulièrement opportune aujourd'hui pour deux raisons historiographiques. D’une part, elle coïncide avec le nouvel intérêt suscité ces dernières années par l'œuvre et la personne de Feijoo au sein des études littéraires, philosophiques et historiques. En témoignent le symposium international réuni à l'Université d'Oviedo en 2014 à l'occasion du 250e anniversaire de sa mort, ainsi que la sortie du second volume de ses CEuvres complètes, plus de 30 ans après la publication du premier en $1981^{4}$. D'autre part, dans le cadre plus large de l'historiographie féministe internationale, il est désormais possible de réexaminer ses écrits à la lumière des analyses qui, au cours des dernières décennies, ont repris et actualisé les connaissances sur la «querelle des femmes» dans son développement historique et ses connexions européennes.

3 Marañón 1962 [1934].

4 III Simposio Internacional sobre el Padre Feijoo. Con la razón y la experiencia: Feijoo 250 años después, Oviedo, les 27 et 28 novembre 2014. Voir aussi Feijoo 2014 et Urzainqui 2003. 


\section{Ce que Feijoo doit à la tradition de la « querelle »}

Benito Jerónimo Feijoo, né en 1676, est l'aîné d'une famille de la moyenne noblesse galicienne. Cependant, sa vocation religieuse et, plus encore, son goût pour les études l'ont conduit à renoncer à son droit d'aînesse (majorat), et à prendre en 1690 l'habit de l'ordre des bénédictins. Il fut professeur de théologie à Oviedo, petite ville de la périphérie nord de l'Espagne, où il vécut la plus grande partie de son existence, de 1709 à sa mort en 1764. Depuis sa cellule du monastère de Saint-Vincent et sa chaire à l'Université, il a produit un intense travail intellectuel de lecture et d'écriture, participant aux débats et à la diffusion des idées de son temps. Ses œuvres constituent le pilier essentiel de la première génération des Lumières espagnoles, et concrétisent l'effort de séparation nette, propre aux Lumières catholiques, du monde de la Révélation - situé au-delà du champ d'action de la critique intellectuelle - de celui de la démonstration empirique et rationnelle. Avec les neuf tomes de son Teatro crítico universal de errores comunes, publiés entre 1726 et 1740, et les cinq volumes de ses Cartas Eruditas y Curiosas (1742-1760)5, œuvres destinées à un assez large public, Feijoo s'est donné pour objectif explicite de combattre les «préjugés » ou «erreurs communes », à la lumière de la raison. Pour ce faire, il a appliqué la critique rationnelle à une grande diversité de sujets: les coutumes et superstitions populaires, l'éducation, la science, l'histoire, la politique ou les relations sociales. Par ses lectures, il était familier des auteurs britanniques et français du $\mathrm{XVII}^{\mathrm{e}}$ et du début du XVIII ${ }^{\mathrm{e}}$ siècles : Newton, Locke, et en particulier Boyle et son empirisme, ainsi que le sceptique Bacon ; il avait aussi lu Descartes, Bayle, Fontenelle, Malebranche, Gassendi et les érudits libertins - La Mothe Le Vayer. Il trouva dans leurs œuvres un appui à son scepticisme intellectuel (mais jamais religieux) qu'il partagea avec d'autres auteurs espagnols de son temps. L'importance de ses écrits, véritables best-sellers du XVIII ${ }^{\text {e }}$ siècle espagnol, résulte de la grande diffusion qu'ils atteignirent avec des tirages de 3000 exemplaires - ce qui était exceptionnel pour l'époque-, suivis de nombreuses

5 Théâtre critique universel des erreurs communes et Lettres érudites et curieuses, œuvres très partiellement traduites en français $(\mathrm{Ndt})$. 
réimpressions ${ }^{6}$. À quoi il faut encore ajouter les éditions clandestines, les impressions séparées, accessibles aux lecteurs plus modestes, et les traductions en plusieurs langues: la Défense des femmes fut traduite en français en 1743 et 1755, en anglais en 1765, 1774 et 1778.

La Défense des femmes constitue le XVI ${ }^{\mathrm{e}}$ discours du premier volume $\mathrm{du}$ Teatro crítico et est reconnu depuis longtemps comme l'un des principaux textes fondateurs du féminisme en Espagne ${ }^{7}$. Partant, on a souvent présenté son auteur comme quelqu'un qui était en avance sur son temps, en faisant l'impasse sur la tradition vieille d'un siècle qui l'avait précédé, et sur le contexte intellectuel espagnol et européen dans lequel son œuvre s'inscrit. Feijoo lui-même, en un certain sens, a contribué à créer son propre mythe de précurseur solitaire en présentant sa défense des femmes comme une entreprise audacieuse de rénovation de la pensée - ce que souligne la citation qui se trouve en exergue de cet article; un point de vue nouveau qui prétend inclure la polémique sur les sexes dans la lutte contre l'erreur en se servant de l'arme de la raison comme seul instrument valable pour combattre le préjugé d'une infériorité féminine. Face à une croyance qu'il dit être enracinée aussi bien dans «l'erreur » populaire que dans l'obscurantisme scolastique, il se méfie des autorités intellectuelles, de son propre aveu presque toutes misogynes, et n’hésite pas à manier l'esprit critique sans craindre de surprendre ni de scandaliser.

Mais, en même temps, cette entreprise que Feijoo évoque comme une aventure pleine de risques n'est pas du tout isolée ni dépourvue de précédents. Nous connaissons bien aujourd'hui la portée qu'a eue dans toute l'Europe la vaste polémique sur les capacités et les penchants moraux, physiques et intellectuels des deux sexes, et particulièrement sur l'infériorité ou l'excellence des femmes, débat que l'historiographie a appelé la «querelle des femmes $»^{8}$. Les études menées au cours des dernières décennies ont démontré l'intensité de

6 Lopez 2003.

7 Ortega 1988 ; Blanco 2010 (édition d'un travail original de 1978). Par ailleurs, les travaux consacrés à la question des femmes depuis la sphère particulière des études sur Feijoo ignorent souvent la tradition européenne de la querelle et les études les plus récentes sur ce sujet, et tendent à proposer par conséquent des interprétations anachroniques et décontextualisées.

8 Zimmerman 2001 ; Viennot 2012. 
la discussion, ses recoupements fréquents avec les préoccupations sociales, intellectuelles et politiques de l'époque - tel le débat sur le mariage et la sexualité, l'affrontement des prêtres et des hommes de lettres laïcs pour la suprématie intellectuelle, la question du gouvernement des femmes ou le choc entre scolastique et philosophie moderne -, son enracinement indéniable dans les milieux de cour et les modalités d'intervention des femmes dans la polémique, comme auteures, mécènes, protagonistes ou lectrices? Ces travaux ont aussi examiné les vastes ramifications internationales de ce débat et l'intense circulation des textes à travers toute l'Europe. Dans la péninsule Ibérique, le débat a débuté très tôt, à partir du XIV ${ }^{\mathrm{e}}$ siècle, dans le cadre des liens étroits qui unissaient la Couronne d'Aragon et l'Italie, et, de là, s'est étendu à la Couronne de Castille où il a trouvé un terreau favorable à la cour des reines Marie d'Aragon et Isabelle de Castille aux XVe et XVI ${ }^{\mathrm{e}}$ siècles ${ }^{10}$. Il se prolongera au siècle suivant pour atteindre son point culminant au XVIII ${ }^{\mathrm{e}}$ siècle ${ }^{11}$.

De fait, Feijoo lui-même reconnait sa dette envers les auteurs, hommes et femmes, qui l'ont précédé, et en vient à donner raison aux femmes qui défendent l'égalité : «Et ainsi, les femmes qui disent que l'âme n'est ni mâle ni femelle peuvent rester sur leur position, car elles parlent juste $»^{12}$. Nous ne pouvons savoir avec certitude à quelles auteures il se référait. L'idée que les âmes n'ont pas de sexe, adaptée de la pensée chrétienne d'inspiration augustinienne, avait été utilisée pour défendre la capacité intellectuelle des femmes par plusieurs écrivains de différents pays entre le XVII e et le début du XVIII siècle. Feijoo se réfère à certaines d'entre elles ailleurs dans son essai - Marie de Gournay, Lucrecia Marinelli- mais aussi à d'autres dont les œuvres ou l'existence ne lui étaient probablement pas connues - telle l'anglaise Mary Astell. En Espagne, cette idée apparaît fort tôt dans le prologue que l'écrivaine castillane María de Zayas avait adressé en

9 Bock \& Zimmermann 1997 ; Rochefort \& Viennot 2013; Haase-Dubosc \& Henneau 2013 ; Dubois-Nayt, Dufournaud \& Paupert 2013.

10 Aichinger et al. 2005 ; Archer 2005 ; Bolufer \& Cabré 2013 ; Bolufer \& Cabré, sous presse.

11 Kitts 1995 ; Bolufer 1998 et 2005 ; Smith 2006 ; Blanco 2010.

12 Feijoo 1778-1779 : 356 . 
1637 «à celui qui lira », en tête de son recueil de récits brefs intitulés Novelas amorosas y ejemplares. Dans ce texte, María de Zayas questionne la misogynie de son temps et construit une défense passionnée de la capacité intellectuelle des femmes, faisant de l'ignorance la clé d'explication de leur soumission, argument que Feijoo développera et qui deviendra le pivot des discussions au Siècle des Lumières :

Et ainsi, la véritable cause du fait que les femmes ne sont point doctes n'est pas un défaut de fond, mais un manque de pratique. Car, pendant notre éducation, si au lieu de nous poser de la batiste sur nos boîtes à couture et des dessins sur nos châssis à broder, on nous donnait des livres et des précepteurs, nous serions aussi aptes aux emplois et aux chaires que les hommes, et peut-être plus subtiles, par notre nature plus froide, car l'entendement est fait d'humidité ${ }^{13}$.

Zayas se situe dans la théorie des humeurs qui, suivant la doctrine galénique, attribue à chaque sexe un certain degré de chaleur et d'humidité ${ }^{14}$ : les hommes sont chauds et secs, les femmes froides et humides; mais elle refuse d'admettre qu'il pourrait en dériver une quelconque limitation de la capacité intellectuelle de ces dernières. Au contraire, elle affirme strictement l'égalité de «l'âme »:

Car si cette matière dont hommes et femmes sommes composés, cet amas de boue et de feu, ou bien cette masse d'esprit et de terre, n'a pas plus de noblesse chez eux que chez nous; si le sang est le même, les sens, les capacités et les organes par où ses effets se font sentir sont les mêmes; [nous avons] la même âme qu'eux, parce que les âmes ne sont ni hommes ni femmes: quel motif fait qu'ils soient savants et puissent prétendre que nous ne pouvons l'être ${ }^{15}$ ?

13 Zayas 2004. "Y así, la verdadera causa de no ser las mujeres doctas no es defecto del caudal, sino falta de la aplicación. Porque si en nuestra crianza, como nos ponen el cambray en las almohadillas y los dibujos en el bastidor, nos dieran libros y preceptores, fuéramos tan aptas para los puestos y para las cátedras como los hombres, y quizá más agudas, por ser de natural más frío, por consistir en humedad el entendimiento ».

14 Maclean 1980.

15 Zayas 2004. "Porque si esta materia de que nos componemos los hombres y las mujeres, ya sea una trabazón de fuego y barro, o ya una masa de espíritus y terrones, no tiene más nobleza en ellos que en nosotras; si es una misma la sangre, los sentidos, las potencias y los órganos, por donde se obran sus efectos, son unos mismos; la 
Il n'est pas possible de savoir si Feijoo avait lu l'œuvre de María de Zayas qu'il ne mentionne jamais. En revanche il cite, admiratif, une autre auteure espagnole un peu antérieure, Oliva Sabuco ${ }^{16}$. Sous ce nom avait paru en 1587 le livre intitulé Nueva filosofía de la naturaleza del hombre [Nowvelle philosophie de la nature de l'homme]. Attribué à Oliva Sabuco dès sa publication, la découverte, au début du $\mathrm{XX}^{\mathrm{e}}$ siècle, du testament manuscrit de son père, Miguel Sabuco, dans lequel il s'en déclarait l'auteur, ouvrit un débat qui n'est pas totalement clos sur son attribution; on a donc affaire soit à un auteur unique, homme ou femme, soit à une possible collaboration entre le père et la fille ${ }^{17}$. En tout cas, ce texte, célèbre à son époque, réédité à trois reprises et diffusé en Europe sous le nom d'Oliva Sabuco, offrait une explication plus équilibrée de la différence des sexes en omettant l'habituelle hiérarchie entre eux. Pour contextualiser la référence de Feijoo, il est important de signaler que la Nueva filosofía - rééditée en 1588 puis de nouveau en 1622, cette fois au Portugal - avait reçu en 1718 les louanges du médecin Vicente Boix y Moliner dans son Hipócrates restaurado [Hippocrate restauré]. Une décennie plus tard, en 1728, un autre médecin, Martín Martínez, chef de file de ce qu'on appelle l'école éclectique et ami de Feijoo, publiera une quatrième édition d'une œuvre qui n'était plus éditée depuis 1622, accompagnée d’un prologue élogieux ${ }^{18}$. Il est fort probable qu'au temps où il préparait cette édition et son introduction flatteuse, Martínez ait partagé avec Feijoo ses impressions et ses réflexions, ce dernier étant de son côté occupé à la rédaction de sa défense des femmes ${ }^{19}$.

De même, parmi les «femmes savantes » du passé et celles de son époque qu'il évoque longuement, Feijoo mentionne Marie de Gournay $^{20}$. Il ne semble pas qu'il ait connu directement ses écrits, dans lesquels - en particulier dans Égalité des hommes et des femmes (1622) et Le grief des dames (1626) - une défense des femmes organisée

misma alma que ellos, porque las almas ni son hombres, ni mujeres: ¿qué razón hay para que ellos sean sabios y presuman que nosotras no podemos serlo ? »».

16 Feijoo $1778: 371-372$.

17 Pomata 2010.

18 Sabuco 1728.

19 Martínez Vidal 1987.

20 Feijoo 1778-1779 : 372. 
autour de l'idée d'une égalité des sexes avait été énoncée pour la première fois ; mais, au moins, l'écho de leur renom avait-il dû lui parvenir. En revanche, on sait qu'il a lu à la Bibliothèque royale de Madrid l'œuvre de Lucrezia Marinella, La nobiltà e l'eccellenza delle donne, co' difetti et mancamenti de gli nomini (1600), car il en qualifie l'auteure de :

docte Vénitienne qui, entre autres œuvres, a composé un livre intitulé : Excellence des femmes, comparée aux défauts et aux vices des hommes, dont le sujet consistait à prouver l'avantage de son sexe sur le nôtre ${ }^{21}$.

Par ailleurs, parmi les savantes qu'il dit admirer figurent aussi la Française Madeleine de Scudéry et la Néerlandaise Anna Maria van Schurmann, des noms tout aussi importants dans cette querelle ${ }^{22}$. Il est significatif que Feijoo fasse non seulement défiler une galerie de femmes savantes, mais s'appuie sur certaines d'entre elles en tant que sources pour la défense de l'égalité, reconnaissant de ce fait leur autorité intellectuelle.

\section{Critique rationaliste et milieux mondains}

Nous pouvons aussi associer Feijoo à un autre courant de pensée, minoritaire à son époque mais pesant d'un certain poids auprès des élites mondaines et cultivées. Celui-ci défendait l'égalité rationnelle et était marqué tant par la philosophie cartésienne que par l'expérience des relations et des échanges intellectuels dans des univers sociaux mixtes. Plongeant ses racines au début du XVII siècle - de Marie de Gournay à Marguerite Buffet - il avait trouvé son expression la plus achevée dans l'œuvre de François Poulain de la Barre (1647-1733)23. On ne dispose pas de preuve que Feijoo ait lu les œuvres de Poulain de la Barre. Le fait qu'il écorche son nom ("Mr. Frelin»), reproduisant l'erreur commise par Pierre Bayle, suggère qu'il en avait une connaissance indirecte, à travers l'information donnée par l'auteur huguenot français du Dictionnaire historique et critique, l'un de

21 Feijoo 1778-1779 : 350. « docta Veneciana, entre otras obras que compuso, una fue un libro con este título: Excelencia de las mujeres, cotejada con los defectos, y vicios de los hombres, donde todo el asunto fue probar la preferencia de su sexo al nuestro ».

22 Feijoo 1778-1779: 373-374 et 380-381.

23 Stuurman 2005. 
ses ouvrages de référence. Cependant ses textes montrent des ressemblances significatives que $j$ 'ai eues l'occasion de mettre en avant dans une étude précédente, dans la mesure où tous deux ont eu, jusqu'à un certain point, des préoccupations et des engagements semblables ${ }^{24}$. Aussi bien Poulain que Feijoo se sont efforcés de se distancier, avec un certain dédain, de la galanterie dont faisaient montre ceux qui se présentaient eux-mêmes comme les « champions des femmes $»^{25}$, préférant prendre la pose du philosophe guidé par une volonté d'objectivité. À partir de leurs positions philosophiques respectives - le premier étant un cartésien convaincu, le second davantage porté vers les courants empiristes - tous deux font usage $\mathrm{du}$ dualisme entre corps et esprit comme d'un instrument pour démontrer l'égalité intellectuelle entre hommes et femmes. Ils s'appuient à la fois sur le raisonnement abstrait et sur la preuve empirique afin d'interroger les différences anatomiques entre les cerveaux masculin et féminin. Ainsi, en limitant la différence sexuelle à quelques caractéristiques corporelles (concrètement celles liées à la reproduction), Feijoo en arrive à la conclusion suivante :

Les femmes ne sont pas formées différemment des hommes pour ce qui est des organes qui servent à la faculté discursive ; elles ne le sont en revanche que pour ceux que la nature a attribués à la propagation de l'espèce ${ }^{26}$.

Argumentation rationnelle et preuve matérielle lui servent ainsi à nier tout fondement naturel à l'infériorité féminine et à affirmer une conception non sexuée de la raison : "l'âme n'est ni mâle ni femelle », écrit-il, en des termes semblables à ceux dans lesquels s'exprimait Poulain - «l'esprit n'a point de sexe $»^{27}$. Ce sont aussi ceux de María de Zayas, un siècle plus tôt ${ }^{28}$.

Les sources sur lesquelles s'appuie Feijoo contiennent aussi d'autres références qui n'ont peut-être pas été assez soulignées par l'historiographie. Parmi elles, Jean-Baptiste Morvan de Bellegarde

\footnotetext{
24 Bolufer 2005.

25 Angenot 1977. En français dans le texte (Ndt).

26 Feijoo 1778-1779 : 359.

27 En français dans le texte $(\mathrm{Ndt})$.

28 Feijoo 1778-1779 : 356.
} 
(1648-1734), figure primordiale de la culture mondaine française et théoricien de l'bonnêteté29, ce modèle de comportement propre à la bonne société de la fin du XVII siècle qui gravitait autour des salons ${ }^{30}$. Cette tradition a pu contribuer, comme l'a récemment avancé Anthony La Vopa, à la défense de l'égalité intellectuelle entre les sexes, dans des proportions plus grandes que ce qu'on avait pu imaginer jusqu'alors. C'est particulièrement le cas de Poulain qui, en combinant ce discours discriminant sur le plan social à la philosophie cartésienne, lui a donné une portée et une radicalité beaucoup plus grandes ${ }^{31}$. Dans ses Réflexions sur la politesse des mours (1698), Bellegarde affirme explicitement l'importance d'établir des relations de mixité et de guider l'existence des dames pour acquérir l'essence du comportement civil. Bellegarde a également écrit un Éloge de l'intelligence des femmes inclus dans ses Lettres curieuses de littérature et morale (1702). Il s'agit d'une réponse fictive écrite par un abbé à une dame qui s'intéressait à la question controversée de l'égalité ou de l'infériorité intellectuelle des femmes, thème fréquent dans les salons et les débats académiques français de la seconde moitié du XVII siècle. Il y défend l'égale aptitude des sexes à exercer n'importe quelle fonction, en reproduisant, en quelque sorte, le mode de la conversation propre à ces cercles. Même s'il est possible qu'il se soit inspiré de Poulain sans le citer, le raisonnement de Bellegarde ne s'appuie pas sur des autorités intellectuelles mais fait appel à sa propre expérience dans le domaine de la mixité des relations sociales, ce pourquoi il assigne aux femmes, en plus de capacités rationnelles identiques aux hommes, une aptitude particulière aux manières et au langage raffinés qui en fait, à son avis, des maittresses en matière de civilité $^{32}$. Deux autres de ses textes ont été traduits en castillan, réunis dans un même volume intitulé El arte de conocer a los hombres, y máximas para la sociedad civil [L'art de connaître les hommes et maximes pour la société civile] (Anvers 1743 et 1755, Madrid 1778 et 1787) qui, avec certaines de

\footnotetext{
29 En français dans le texte, souligné par l'auteur (Ndt).

30 France 1992 : 55-56.

31 La Vopa 2010.

32 Morvan de Bellegarde 1707 : 151.
} 
ses œuvres éditées en français, fut de lecture courante dans le public espagnol cultivé du XVIII siècle $^{33}$.

Feijoo utilise Bellegarde à l'appui de son affirmation selon laquelle: "L'esprit des femmes n'est de nulle façon inférieur à celui des hommes pour aucune des sciences, arts ou emplois $»^{34}$. Comme Bellegarde et aussi Poulain, il insiste sur le fait que les recherches anatomiques démontrent l'égalité des cerveaux féminin et masculin. Comme eux, il dénonce aussi la partialité de lois élaborées pour les seuls hommes; ceux-ci se trouvant juges et parties, ils ont exclu les femmes de l'accès aux charges et aux offices. Feijoo attribue cette « usurpation» non pas à leur incapacité, mais à la coutume et aux préjugés. Bien qu'il reconnaisse n'avoir pas consulté Bellegarde directement mais le connaitre à travers ses sources de référence principales sur la vie intellectuelle française, il lui attribue cependant une autorité pour se prononcer sur cette question, autorité basée précisément sur son expérience de la fréquentation de la bonne société (et, implicitement, des dames) :

Le second témoin est ce Français très érudit, l'abbé de Bellegarde, qui fut aussi homme de cour et qui connut très bien le monde du grand théâtre de Paris. [...] Je n'ai pas rencontré cet auteur mais il est cité à ce sujet dans les mémoires de Trévoux au mois d'avril de l'an $1702^{35 .}$

\section{Une argumentation originale?}

Ainsi donc, la construction rhétorique de la Défense des femmes, ses stratégies et arguments, constituent un mélange de tradition et de nouveauté. La défense de l'excellence morale des femmes chez Feijoo est plutôt conventionnelle; il fait une comparaison des qualités qu'il considère innées et spécifiques à chacun des sexes, où la balance penche du côté des femmes : aux attributs «masculins» de robustesse, de

33 Bolufer 2013.

34 Feijoo $1778:$ 355, «El espíritu de las mujeres no es en alguna manera inferior al de los hombres para cualquiera de las ciencias, artes o empleos ».

35 Feijoo 1778-1779 : 355, «El segundo testigo es el eruditísimo Francés el Abad de Bellegarde, hombre también áulico, y que conoció bien el mundo en el gran Teatro de París. [...] No he visto a este Autor, pero le citan sobre este asunto los de las Memorias de Trevoux en el mes de Abril del año de $1702 »$. 
constance et de prudence, il oppose les vertus «féminines » de beauté, de docilité et de pudeur ou, selon ses propres mots, de «vergogne». En ce sens, Feijoo et ses partisans s'affrontent directement à un discours misogyne des plus intraitables, d'origine ecclésiastique, qui soutient que les hommes sont capables d'un plus grand contrôle de leurs passions et de leurs appétits, tandis que les femmes, lascives par nature, s'efforcent de les séduire et de les écarter du droit chemin par leur beauté, façade trompeuse d'un intérieur pervers.

Notre bénédictin admet aussi, bien qu'avec un certain degré de scepticisme, les principes humoraux du galénisme qui avaient dominé la médecine occidentale depuis le Moyen-Âge et qui attribuaient aux deux sexes des tempéraments opposés. Il se situe ainsi dans la droite ligne de ce qu'on a appelé le « féminisme galénique », qui débute à la Renaissance et du côté duquel on peut, dans une certaine mesure, ranger la position de María de Zayas ainsi que celle d'Oliva Sabuco ${ }^{36}$, puisqu'il refuse que ces caractéristiques limitent la capacité intellectuelle des femmes. Feijoo était très familier des débats de la science médicale de son époque, et il participa activement à certains d'entre eux - par exemple, à celui qui eut lieu autour de ce qu'on appela la «médecine sceptique». Cette expérience lui permit de prendre conscience du fait que les raisonnements présentés par les scientifiques, sur lesquels s'appuyaient les affirmations de l'infériorité intellectuelle des femmes, constituaient des arguments ad hoc pour justifier l'inégalité. À ses yeux, ces arguments manquaient non seulement de fondement empirique ou anatomique solide, mais on pouvait en tirer des conclusions totalement opposées ${ }^{37}$ :

ces causes physiques, on sait bien que chacun les cherche et les retient à sa guise, après que par l'expérience on est ou on pense être assuré de leurs effets ${ }^{38}$.

De même Feijoo montre-t-il, en anti-scolastique convaincu, son mépris ironique pour ceux pour lesquels le simple nom d'Aristote était synonyme de vérité. Il insiste également sur le fait que les

\footnotetext{
36 Pomata 2013.

37 Bolufer 2009 ; Pomata 2013.

38 Feijoo 1778-1779: 351, «Estas causas físicas ya se sabe que cada uno las busca, y señala a su modo, después que por la experiencia está, o se juzga asegurado de los efectos ».
} 
principes aristotéliciens ne sont pas des preuves de l'infériorité des femmes. Par surcroît, il met en question une autre justification de l'inégalité des sexes qui commençait alors à remplacer les idées scolastiques classiques: celle énoncée par le cartésien Nicolas Malebranche (De inquirenda veritate, 1674), d'une plus grande sensibilité des femmes - ou de l'excessive mollesse de leurs fibres cérébrales cause de leur infériorité en matière d'acquisition du savoir ${ }^{39}$. Feijoo réfute cette théorie par des arguments rationnels et empiriques. D'une part, il questionne leur base expérimentale - «J'ai lu deux anatomistes qui n'en disent mot $»^{40}$. D'autre part, il lui semble contradictoire de vouloir attribuer à la supériorité sensible des fibres féminines une moindre capacité rationnelle alors que cette caractéristique pourrait être considérée comme un avantage du point de vue de l'épistémologie empiriste pour laquelle toute connaissance a pour origine les impressions reçues à travers les sens :

Donc, les fibres du cerveau des femmes étant plus flexibles que dans celui des hommes, celles-ci devront former des images plus grandes et plus distinctes et par conséquent mieux percevoir les objets ${ }^{41}$.

Alors que sa réfutation de la scolastique à base aristotélicienne s'adresse à une philosophie encore en vigueur dans l'enseignement universitaire (mais déjà bien mis en question dans les milieux intellectuels espagnols), son désaccord avec Malebranche anticipe la direction que vont prendre les idées sur la différence des sexes dans le nouveau contexte de la « culture de la sensibilité » propre à la seconde moitié du XVIIIe siècle.

Enfin, Feijoo recourt au catalogue des «femmes savantes» des siècles précédents et le complète par des exemples tirés de l'histoire récente, ordonnés par lieux d'origine - les "Espagnoles savantes", « France », « Angleterre », etc. - pour prouver que toute nation qui se prétend cultivée se doit d'exhiber son propre panthéon de femmes

39 La Vopa 2010 : 69-70 ; pour une interprétation de la pensée de Malebranche très différente de la mienne, voir Hamerton 2008 et 2010.

40 Feijoo 1778-1779 : 366, «Dos Anatómicos he leído que no dicen palabra de eso ».

41 Feijoo 1778-1779 : 367, « Uego siendo en el cerebro de las mujeres más flexibles las fibras que en el de los hombres, formarán aquellas mayores, y más distintas las imágenes, y por consiguiente percibirán mejor los objetos ». 
illustres. Particulièrement connaisseur de la culture française, il est tout spécialement frappé par l'éclosion d'érudites et d'écrivaines au $\mathrm{XVII}^{\mathrm{e}}$ et au début du XVIII ${ }^{\mathrm{e}}$ siècle, depuis Marie de Gournay et Madeleine de Scudéry jusqu'à l'helléniste Anne Dacier ; cela l'amène à établir des comparaisons peu flatteuses pour son propre pays :

Les Françaises savantes sont fort nombreuses car les femmes ont davantage de possibilités en France et, je le crois aussi, plus de libertés pour étudier ${ }^{42}$.

Ce fait lui sert à renforcer ses arguments sur l'importance vitale de l'éducation et des habitudes quand il s'agit de stimuler ou d'inhiber le potentiel naturel, égal, en principe, entre hommes et femmes : si les Espagnoles, selon lui, n'ont pas été si nombreuses à faire montre de leurs talents pour les lettres, c'est à cause de leur réclusion domestique et de la rareté des occasions qui leur sont données de fréquenter des personnes cultivées. Feijoo invalide ainsi les arguments de ses adversaires, qui confondaient l'ignorance des femmes avec un manque d'aptitudes intrinsèques. Pour lui, au contraire, l'inégalité sociale entre les sexes dans l'accès au savoir ne reflète pas la hiérarchie naturelle. Elle fait violence à l'égalité originelle d'une manière que seuls ceux qui regardent la réalité à travers la lentille déformante du "préjugé » peuvent confondre avec la véritable nature des choses. Un raisonnement que Feijoo appliquera aussi à l'égalité naturelle des êtres humains dans différentes parties du monde, contre l'idée déterministe de "caractères nationaux» ou de supposées différences morales et intellectuelles entre les différentes "races», dans son essai Mapa intelectual y cotejo de naciones ${ }^{43}$.

\section{Polémiques, contradictions, protections}

La Défense des femmes suscita, comme d'autres essais de Feijoo, une ardente polémique qui donna lieu en quelques années à un total de 29

42 Feijoo 1778-1779 : 373, «Las Francesas sabias son muchísimas, porque tienen más oportunidad en Francia, y creo que también más libertad para estudiar ».

43 [Carte intellectuelle et comparaison des nations], discours XV du tome II de son Teatro crítico publié pour la première fois en 1728. 
publications ${ }^{44}$. Parmi les défenseurs de Feijoo, le médecin Martín Martínez prit appui sur l'autorité que lui conféraient sa formation et son expérience scientifique :

Au moins, moi, en tant que professeur d'anatomie, je peux dire que l'organisation qui diversifie les deux sexes n'étant pas l'instrument des pensées, et l'homme et la femme étant aptes à la fabrique de la pensée - seul siège et entrepôt des idées - je dois croire que, dans l'aptitude aux sciences, les emplois ne sont pas inégaux vu que les organes ne sont pas différents ${ }^{45}$.

À l'inverse, les défenseurs des thèses les plus conservatrices accusèrent Feijoo d'altérer l'ordre naturel et providentiel qui justifiait, du fait de l'infériorité innée des femmes, leur nécessaire subordination à l'intérieur de la famille et de la société, en subvertissant les relations de pouvoir entre les sexes et, par extension, toutes les hiérarchies que ces relations symbolisaient de façon plus profonde. C'est ce que Santarelli affirmait de façon éloquente :

Le père [Feijoo] veut que les femmes répliquent à leurs maris, se reconnaissent leurs égales et ne leur accordent pas de supériorité [...] ; par ma foi, le père [Feijoo] a trouvé le moyen de ruiner toutes les républiques, catholiques ou non catholiques, car le vassal peut dire au prince qu'il n'a pas à lui obéir ${ }^{46}$.

Cependant, Feijoo et ses partisans n'avaient pas l'intention de remettre en cause l'ordre social, qu'ils pensaient établi par la Providence. Suivant cet ordre, les femmes devaient reconnaitre et respecter l'autorité masculine aussi bien dans l'univers domestique que sur le terrain politique. Ils voulaient seulement faire reposer cet ordre sur des bases différentes. Pour Feijoo, la nécessaire soumission

44 Bolufer $1998: 27-59$; Smith $2006: 17-39$.

45 Martínez 1726 : 18, «A lo menos, yo como profesor anatómico puedo decir que no siendo la organización que diversifica los dos sexos instrumento de los pensamientos, y conviniendo hombre y mujer en la fábrica del pensamiento (única silla y emporio de las ideas), debo creer que en la aptitud para las Ciencias no son desiguales los oficios, pues no son diferentes los órganos ».

46 Santareli [s.d.] : 35, «El Padre quiere que las mujeres repliquen a sus maridos, se reconozcan por iguales a ellos y no les confiesen superioridad [...] ; a fe mía, que el Padre había dado en el modo de arruinar todas las repúblicas, católicas y no católicas, porque el vasallo podía decir al príncipe que no le debía obedecer ». 
des femmes ne dérive pas de leur infériorité mais du châtiment d'Ève pour son péché, ce qui est un précepte de droit positif - divin -, mais en aucun cas de droit naturel. Reconnaitre l'égalité naturelle des sexes constitue donc un triomphe de la vérité sur le préjugé, mais ne porte pas préjudice à la suprématie masculine. Cela peut même la renforcer, éliminant le conflit au bénéfice des mœurs, car les femmes qui seront convaincues de leur propre valeur et seront respectées par leurs maris, seront chastes et obéissantes de leur propre volonté.

Pour comprendre la pensée de Feijoo dans sa complexité, il est cependant nécessaire de prendre en considération non seulement l'essai sur la Défense des femmes, en tant que tel, mais aussi l'ensemble de son œuvre, riche, diverse et parfois contradictoire puisque la campagne qu'il mène contre les «préjugés » n'est pas toujours rigoureuse quant à la condition et la «nature» des femmes. Par exemple, dans son discours intitulé Virtud aparente $[V \text { ertu apparente }]^{47}$, Feijoo attribue aux femmes une plus grande tendance à une certaine forme d'hypocrisie qui consiste à lui faire prendre la simple apparence de la vertu :

Une femme, parce qu'elle est chaste, juge qu'elle a rempli toutes les cases de la vertu ; ou du fait qu'elle possède cette seule vertu, elle juge que tous les autres vices lui sont autorisés. Ainsi, ayant donné toutes les preuves en cette matière, elle peut être arrogante, envieuse, impatiente ${ }^{48}$.

De même ne se montre-t-il pas toujours convaincu de l'aptitude féminine à la retenue qu'il avait exaltée avec tant d'emphase dans sa Défense des femmes. Dans un passage de l'essai intitulé La ociosidad desterrada, y la milicia socorrida [L'oisiveté bannie et la milice secourue ${ }^{49}$, alertant sur les vices auxquels sont poussés les pauvres du fait de leur misère vols, faux témoignages - il poursuit : «Ces vices sont communs aux pauvres des deux sexes. Chez les femmes s'y ajoute la lascivité $»^{50}$. Bien

47 Publié dans le tome IV du Teatro crítico.

48 Feijoo 1778-1779: IV, I, 9, «Una mujer con ser casta, juzga que tiene llenos todos los números de la virtud; ó con poseer esta virtud sola, juzga que le son lícitos todos los demás vicios. Así, teniendo bien hechas las pruebas en esta materia, puede ser arrogante, envidiosa, impaciente ». Voir aussi Gomis 2009.

49 Discours XIII du tome VIII.

50 Feijoo 1778-1779 : VIII, XIII, 438-439, « Estos vicios son comunes a los pobres de uno, y otro sexo. En las mujeres se agrega el de la lascivia ». 
qu'ici Feijoo se réfère concrètement aux femmes dont l'existence est conditionnée par la misère, affirmer si catégoriquement que la lascivité est un vice féminin va à l'encontre de son apologie antérieure du naturel pudique de ce sexe sur lequel il avait fait reposer l'idée de sa supériorité morale. Il se rapproche des lieux communs les plus arbitraires de la misogynie savante aussi bien que populaire.

C'est au sein même de son discours, dans la défense qu'il fait du caractère asexué de la raison que l'on remarque le plus clairement ses doutes et ses contradictions, à la lecture de deux de ses écrits en particulier. Ainsi, dans l'essai intitulé Demoníacos (discours VI du tome VIII) où il mène campagne contre la croyance en la possession diabolique qu'il considère dans la plupart des cas comme une superstition et le produit de l'ignorance ${ }^{51}$, Feijoo attribue aux femmes un plus grand penchant aux fausses possessions, du fait qu'elles ont « un cerveau plus faible et une imagination plus vive, qualités qui facilite chez elles la croyance qu'elles sont possédées ${ }^{52}$. Par ailleurs, dans une lettre du 26 février 1749 qu'il adresse à Ana María Moscoso de Prado - relation sur laquelle je reviendrai plus loin - en remerciement de la dédicace d'un de ses poèmes (romance), Feijoo louait la qualité de la pièce, y voyant, de manière assez conventionnelle, une marque exceptionnelle de talent; au point, ajoutait-il, qu'il semblait difficile de croire que ce fût l'œuvre d'une femme. Dans ce texte, écrit plus de vingt ans après la publication de sa célèbre Défense des femmes, le bénédictin essaie de concilier sa conviction de l'égalité des sexes dans certains milieux intellectuels - particulièrement raffinés - avec la différence - et implicitement, l'inégalité - qui règne dans d'autres milieux. Ainsi affirme-t-il :

Bien que du côté de l'esprit et de la subtilité, je tienne toujours ferme qu'il n'y a aucune inégalité entre les deux sexes, il n'en va communément pas de même pour ce qui est de l'énergie, de la force ou de la hardiesse du génie, car j'ai observé que, même dans les œuvres de l'esprit, le beau sexe se ressent de la faiblesse de son tempérament; et jusqu'alors je n'ai

51 Tausiet 2006.

52 Feijoo 1778-1779: VIII, VI, 143-144, «ser de cerebro más débil y más viva imaginación, cualidades que les facilitan el créer ellas mismas que están endemoniadas ». 
pas vu d'exception à cette règle, si ce n'est celle que vous me présentez aujourd'hui, Madame ${ }^{53}$.

Il convient de nuancer légèrement cette affirmation, qui, à première vue, entre en contradiction flagrante avec la défense de l'égalité intellectuelle soutenue par Feijoo dans son essai de 1726, et qu'il a réaffirmée tout au long de la polémique qui l'a opposé à ses détracteurs misogynes. Il est vrai que le registre courtois de la lettre de remerciements impose une certaine rhétorique de l'éloge, qui conduit Feijoo à pousser à l'extrême le compliment qu'il fait à la dame. Il la présente, de manière classique, comme une exception à son sexe, ce qu'avaient fait avant lui d'innombrables galants mis en situation de reconnaittre le talent et la capacité intellectuelle ou créative d'une femme. Cette manière de présenter les choses, dans son cas, contraste avec les portraits des femmes savantes, inclus dans le discours XVI du premier tome du Teatro critico, qu'il présentait moins comme des exceptions que comme les preuves vivantes de l'aptitude des femmes dans toutes les sphères du savoir. Il est vrai aussi que le terme "tempérament» avait au XVIII siècle plusieurs significations, qui comprenaient la combinaison des humeurs à l'intérieur du corps selon le système galénique, mais aussi, dans une certaine mesure, le caractère ou disposition d'esprit, parmi les déterminants hérités et les manières acquises par l'éducation et la coutume. On pourrait ainsi en tirer argument pour dire que Feijoo ne l'utilisait pas ici au sens purement déterministe, biologique, et qu'il se référait aux manifestations concrètes de la production intellectuelle féminine plutôt qu'aux aptitudes potentielles de ce sexe. Cependant, même en acceptant ces nuances, il est difficile de ne pas voir dans sa lettre un rectificatif de sa ferme défense antérieure de l'égalité. Ainsi un net contraste s'établit entre ce texte de Feijoo et ceux consacrés à l'égalité intellectuelle des sexes dans sa Défense des femmes, ce qui

53 Macías 1932: 328, "Aunque por parte de la agudeza e ingeniosidad, estoy siempre firme en el concepto de que no hay desigualdad alguna entre los dos sexos, no es así por lo común en cuanto a la energía, fuerza o valentía del numen, en lo que he observado hasta ahora que aun en las obras mentales se resiente el bello sexo de la debilidad de su temperamento; y hasta ahora no he visto otra excepción a esta regla, que la que se me presenta en Vmd [Vuestra Merced] ». 
démontre que les lieux communs les plus arbitraires ont pu influer sur lui alors même qu'il se présentait comme le porte-drapeau de la lutte contre les préjugés et les idées reçues. Les paradoxes de Feijoo illustrent ainsi la difficulté de penser la différence des sexes en termes strictement rationalistes.

À un autre niveau d'analyse, l'image romantique du génie solitaire de Feijoo s'estompe si on le replace dans le cadre des relations sociales et politiques dans lequel il évoluait. En effet, Feijoo ne fut pas l'ermite isolé qui lisait, pensait et écrivait dans sa cellule sous la seule impulsion de sa propre raison. Au contraire, il s'est montré tout aussi conscient $\mathrm{du}$ poids de l'opinion publique que critique face au «vulgaire» - au sens culturel et pas simplement hiérarchique du mot, le "vulgaire» étant entendu comme opinion vulgaire, pleine de préjugés, produite soit par l'ignorance, soit par une formation archaïque de type scolastique. À ce thème, il a consacré un ouvrage spécifique, intitulé $L a$ voz del pueblo [La voix du peuple], et y a fait de nombreuses références dans certains de ses autres textes. Il fut particulièrement habile à se connecter à de larges publics auxquels il s'adressait explicitement et avec lesquels il entamait dans ses écrits une relation dialogique : ainsi dans les essais du Teatro crítico et surtout dans le registre plus souple et informel de ses Cartas eruditas y curiosas ${ }^{54}$. Il participa intensément aux polémiques intellectuelles et sociales de son temps sur les thèmes les plus variés : de l'histoire, la philologie et la science à la politique. Ce fut un homme au caractère affable, qui entretint durant sa longue existence un réseau dense de relations sociales, cultivées au fil de réunions qui se tenaient dans sa cellule du monastère et dans les maisons où il était invité, ainsi qu'à travers une très abondante correspondance, dont on ne conserve malheureusement qu'une petite partie. Il eut beaucoup de relations amicales tant à Oviedo qu'ailleurs, essentiellement masculines, ce qui est logique étant donné son statut d'ecclésiastique et d'érudit. Parmi ses amis, on signalera, du fait de leur influence sur le monde des lettres, son frère en religion Martin Sarmiento, bénédictin lui aussiintellectuel d'une vaste et profonde érudition, propriétaire d'une des plus importantes bibliothèques de son époque - et les journalistes du Diario de los Literatos [Journal des hommes de lettres].

54 Urzainqui 2014. 
Cependant, son cercle de relations comprenait aussi quelques femmes, parmi lesquelles certaines étaient nobles et éclairées. Ana Moscoso de Prado, déjà citée, aristocrate galicienne très liée à sa famille, lui adressa en 1749, alors que Feijoo était déjà âgé et jouissait d'une large renommée, un poème de louanges auquel le moine répondit par une lettre de remerciements : les deux textes, même s'il sont restés dans la maison familiale des Feijoo sous forme manuscrite jusqu'à la fin du XIX ${ }^{\mathrm{e}}$ siècle, semblent avoir été destinés à circuler dans leurs réseaux, au bénéfice du prestige social et intellectuel tant de la dame que de l'ecclésiastique ${ }^{55}$. Dans ses vers, Ana Moscoso loue la figure et l'œuvre de Feijoo, dont elle se dit admiratrice, en même temps qu'elle déploie sa propre érudition classique. Elle s'adresse au bénédictin depuis une position sociale qui associe la déférence due à une figure consacrée du paysage intellectuel espagnol, et une certaine familiarité complice du fait de sa double condition d'ami de la famille et de confrère dans le monde des lettres. Certains indices montrent que Feijoo a aussi entretenu une correspondance avec d'autres femmes jusqu'alors non identifiées; il adresse trois de ses Cartas curiosas à trois d'entre elles - la septième du I $^{\text {er }}$ tome, la seizième du tome III et la première du tome IV.

Feijoo a également entretenu de très bonnes relations avec le pouvoir politique. Il a bénéficié de la protection des Bourbons, de Philippe V à Charles III, de celle de leurs ministres - Patiño, Campillo, Ensenada, Carvajal, Wall, etc. - et de leurs confesseurs P. Rávago. De fait, c'est grâce à un ordre du roi Ferdinand VI en juillet 1750 que prirent définitivement fin les polémiques autour de l'œuvre de Feijoo. Geste d'autorité inédit, l'interdiction de toute expression publique de désaccord avec l'auteur le plaçait sous la protection royale. L'acte a été qualifié par d'autres auteurs de frein illicite à la liberté d'opinion mais son destinataire l'a apprécié. Il en a

55 Macías (dir.) 1932. L'érudit Marcelo Macías fit connaitre ces textes inédits le 9 septembre 1887, au cours d'une cérémonie religieuse célébrée en la cathédrale d'Orense à l'occasion de l'inauguration d'un monument érigé à la mémoire de Feijoo. Ana Moscoso était voisine et amie de Plácido Feijoo Montenegro, frère cadet de Feijoo et héritier du droit d'aînesse, après la renonciation de Benito, et des biens qui lui étaient attachés (majorat) dans le hameau de Casdemiro, paroisse de Santa María de Melias, dans la province d'Orense (Galice). 
exprimé de la gratitude en dédiant la réédition de 1753 du troisième volume de ses Cartas eruditas (1745) au roi, et le quatrième volume de cette même œuvre, édité aussi pour la première fois en 1753, à son épouse, la reine Barbara de Bragance $(1711-1758)^{56}$. Dans cette seconde dédicace, écrite quatre ans seulement après la lettre à Ana Moscoso de Prado, Feijoo rappelle le fort rejet que sa défense des femmes avait provoqué dans certains milieux intellectuels :

L'acharnement à établir l'égalité intellectuelle des deux sexes, en défendant l'aimable et le faible de l'injure que généralement ou quasi généralement on lui faisait en lui refusant cette égalité. Ô combien de sarcasmes cette noble entreprise m'a-t-elle attirés !57

Il y présente la figure de la reine comme la meilleure preuve vivante de la capacité intellectuelle des femmes. Dame cultivée, Barbara de Bragance avait apporté en Espagne le raffinement de la cour portugaise. Elle parlait six langues et possédait une solide formation qu'on peut apprécier, entre autres signes, à sa bibliothèque personnelle, vaste et bien pourvue ${ }^{58}$. Elle fut mécène d'artistes et de musiciens - elle-même composait et fut une bonne interprète - et, fait significatif, elle se préoccupa de l'amélioration de l'éducation des jeunes filles de la noblesse de cour en fondant le couvent royal de la Visitation qui offrait une formation plutôt laïque et ouverte : elle se fit enterrer dans la chapelle de cette institution. Feijoo intègre dans ses louanges à la reine, à côté d'autres qualités plus conformes à ce qu'on trouve dans tout éloge aux souverains (magnanimité, piété, prudence, etc.), une reconnaissance explicite de ses capacités et de sa formation dans différents domaines du savoir :

Mais l'opposition que j'ai reçue de ce côté, si elle ne s'est pas entièrement dissipée, a beaucoup diminué depuis que Votre Majesté a fait voir au monde l'extraordinaire capacité dont le Ciel l'a dotée. Je pense que la plupart se tairont, ceux qui, seulement parce qu'ils sont hommes, prétendent à la supériorité de leurs talents sur ceux de l'autre sexe, en

56 Feijoo 1774 : II-XV ; Franco 2005.

57 Feijoo 1774 : VIII, «El empeño de establecer la igualdad intelectual de los dos sexos; vindicando el amable, y débil de la injuria, que generalmente, o casi generalmente se le hacía en negarle esta igualdad. ¡Oh, cuántos sarcasmos me atrajo esta noble empresa!».

58 Arias \& Franco 2012. 
voyant chez une Reine l'intelligence de six langues, quand eux arrivent à peine à comprendre médiocrement celle de leur naissance. Je pense que la plupart se tairont, presque inaptes qu'ils sont à toute science ou art, sans autre titre que celui de leur sexe, et prétendant au même avantage, quand ils verront une Reine qui, par-dessus tant de capacités, apportées par une excellente éducation, possède intelligiblement toutes les habiletés [nécessaires à] la Musique, en tant que science et en tant qu'art ; c'est-àdire, la théorie et la pratique. Je pense que bientôt se tairont ceux qui, sans d'autre titre que celui de leur virilité, aspirent à cette préférence, voyant une Reine, qui, dans la conversation, manie avec un juste critère les catégories historiques, politiques et morales; et même quand interviennent des ecclésiastiques, elle use avec grand à propos, en même temps que discrète mesure, des extraits de l'Ecriture alors qu'eux ont toutes les peines à sortir des sujets communs et vulgaires ${ }^{59}$.

Feijoo profite ainsi de l'épître dédicatoire pour remercier les monarques de leur appui, insistant dans le même temps sur l'idée que la croyance en l'infériorité intellectuelle des femmes, dépourvue de fondement, n'est que le produit de l'ignorance: «Seuls les ineptes parlent et se lamentent indignement de la rationalité des femmes ${ }^{60}$.

59 Feijoo 1774 : VIII-IX, «Mas la oposición que padecí en esta parte, si no se desvaneció enteramente, se disminuyó mucho desde que V.M. hizo ver al mundo la extraordinaria capacidad de que la dotó el Cielo. Ya discurro que callarán los muchos, que, sólo a título de varones, pretendían superioridad de talentos sobre el otro sexo, viendo en una Reina la inteligencia de seis lenguas, cuando ellos apenas aciertan a explicarse medianamente en la nativa. Discurro que ya callarán los muchos, que, siendo casi inhábiles para toda ciencia, o arte, sin otro título que el de su sexo, pretenden la misma ventaja; al ver una Reina, que, sobre otras habilidades, que le comunicó una excelente educación, comprehensivamente posee todos los primores de la Música, en cuanto ciencia, y en cuanto arte; esto es, la teórica, y la práctica. Discurro que ya callarán los que, sin más título que el de su varonía, aspiran a esa preferencia, viendo una Reina, que en la conversación maneja con una justa crítica especies históricas, políticas, y morales; y aun cuando intervienen personas eclesiásticas, usa con gran propiedad, al mismo tiempo que con discreta parsimonia, de los lugares de la Escritura; no sabiendo ellos salir de los asuntos más vulgares, y comunes ».

60 Feijoo 1774 : IX-X, «solo los ineptos hablan, y sienten indignamente de la racionalidad de las mujeres ». 
Comme on l'a vu dans les lignes qui précèdent, il est possible de proposer une vision du personnage de Feijoo qui soit moins héroïque et qui le replace dans son contexte historique - social, intellectuel et même politique - ainsi que dans son environnement intellectuel, espagnol et européen. En ne limitant pas la lecture de son œuvre à son essai sur la Défense des femmes, on peut mesurer les ambiguïtés et les paradoxes de sa pensée sur la nature et la portée de la différence des sexes, ainsi que ses résistances, parfois inconscientes, à la reconnaissance de l'égalité. Une telle réinterprétation permet aussi de comprendre comment sa position s'est appuyée tout autant sur une lecture personnelle de la tradition de la «querelle des femmes » que sur une observation de la participation passée et présente des femmes à la culture et à la société. Loin d'être « en avance sur son temps » et, par conséquent, une figure isolée, il n'a pas construit son argumentaire dans la solitude, mais dans un dialogue critique avec ses prédécesseurs et ses contemporains. C'est particulièrement vrai pour celui qu'il a mené avec les auteurs femmes qui avaient, avant lui, défendu l'égalité des sexes en apportant leurs contributions, avidement lues et passionnément discutées, à un débat ardent. La vaste diffusion de son œuvre et le renom qu'elle a acquis eurent une influence déterminante au moment même où émergeait l'opinion publique. Sa défense des femmes a contribué à faire tomber en désuétude les arguments traditionnels de la misogynie dans les cercles intellectuels éclairés. À partir de ce moment-là, tous ceux qui prétendaient avoir un esprit ouvert et des idées modernes, n'osaient plus répéter - au moins en public et par écrit - les lieux communs sur l'infériorité des femmes. Il sera désormais assez souvent fait référence à ces conceptions comme à des préjugés dépassés, propres à des époques lointaines et encore obscures. Dans la deuxième moitié du XVIII ${ }^{e}$ siècle, ceux qui aborderont, en gens des Lumières, cette question si polémique invoqueront le nom de Feijoo comme un talisman, et affirmeront - parfois de manière plus rhétorique que pleinement convaincue - que les femmes ne sont pas inférieures aux hommes en dignité ni en capacité. Ils ajouteront néanmoins parfois que la relation entre les unes et les autres relève d'une «complémentarité » naturelle. On ajoutera enfin que la pensée de Feijoo a circulé dans d'autres pays que l'Espagne, où elle a irrigué la 
discussion sur la différence des sexes, discussion qui, comme les études récentes le montrent, n'a cessé, au XVIII ${ }^{\mathrm{e}}$ siècle et au-delà, d'être un débat ouvert ainsi qu'un champ commun et transnational, où circulaient les idées, les arguments et les références intellectuelles.

Traduit du castillan par Rudy CHAULET

\section{Bibliographie}

II Simposio sobre el Padre Feijoo y su siglo, 1981, Oviedo, Instituto Feijoo de Estudios del Siglo XVIII.

Aichinger Wolfram, BIDwell-STEINER Marlen, BÖSCH Judith \& Eva CESCUTTI (eds), 2003, The Querelle des Femmes in the Romania. Studies in honour of Friederike Hassauer, Vienne, Turian und Kant.

ANGENOT Marc, 1977, Les Champions des Femmes. Examen du discours sur la supériorité des femmes, 1400-1800, Montréal, Presses de l'Université du Québec.

ARCHER Robert, 2005, The Problem of Woman in Late-Medieval Hispanic literature, London, Tamesis Books.

Arias De SAAVEDra Inmaculada \& Gloria FranCO, 2012, «Lecturas de mujeres, lecturas de reinas. La biblioteca de Bárbara de Braganza », in Inmaculada ARIAS DE SAAVEDRA (dir.), Vida cotidiana en la España de la Ilustración, Granada, Universidad de Granada, p. 505-550.

BIDWELL-STEINER Marlen, 2009, Große Welt - kleine Welt - Verkehrte Welt: Die philogyne Naturphilosopbie der Renaissance-Denkerin Oliva Sabuco de Nantes y Barrera, Innsbruck, Studien Verlag.

BLAnco Oliva, 2010, La Polémica feminista en la España ilustrada. La "Defensa de las mujeres" de Feijoo y sus detractores, Ciudad Real, Almud.

Bock Gisela \& Margarete ZimmermanN (dir.), 1997, Die europäische Querelle des Femmes. Gescblechterdebatten seit dem 15. Jahrbundert, Stuggart/Weimar, Verlag J.B. Metzler.

BOLUFER Mónica, 1998, Mujeres e Ilustración. La construcción de la feminidad en la España del siglo XVIII, Valencia, Institució Alfons el Magnànim.

-, 2005, «Neither Male, Nor Female: Rational Equality in the Spanish Enlightenment », in Barbara TAYLOR \& Sarah KNOTT (eds), Women, Gender and Enligbtenment, London, Palgrave, p. 389-409.

—, 2009, "Medicine and the Querelle des Femmes in Early Modern Spain", Medical History. Supplements, 29, p. 86-106. 
—, 2013, "De la cortesía a la civilidad: modelos en tensión», in José MARTíNeZ MiLlán, Concepción CAMARERO \& Marcelo LuZZI (coord.), La Corte de los Borbones: crisis del modelo cortesano, vol. III, Madrid, Ediciones Polifemo, p. 1439-1464.

_, 2015, «La querelle des femmes en Espagne : bilan sur l'histoire d'un débat (1400-1800) », in Marie-Élisabeth HENNEAU, Éliane VIENNOT \& Rotraud VON Kulessa (dir.), Revisiter la Querelle des femmes. Discours sur l'égalité/ inégalité des sexes en Europe, de 1400 aux lendemains de la Révolution, Saint-Étienne, Publications de l'Université de Saint-Étienne, p. 31-46.

BOlufer Mónica \& Montserrat CABRÉ (dir.), 2013, «La Querella de las Mujeres: nuevas perspectivas historiográficas ", Arenal. Revista de historia de las mujeres, 20/2, p. 235-341.

CrAveri Benedetta, 2002, L'Áge de la conversation, Paris, Gallimard.

Dubois NAYT Armel, DUfOURnAUd Nicole \& Anne PAUPERT, 2013, Revisiter la querelle des femmes, vol. 3, Discours sur l'égalitél inégalité des sexes, de 1400 à 1600, Saint-Étienne, Publications de l’Université de Saint-Étienne.

FEIJOO Benito Jerónimo, 1774, Cartas eruditas y curiosas. Nueva impresión, Madrid, Pedro Marín, t. IV [disponible en ligne : http:// filosofia.org/bjf].

—, 1778-1779, Teatro Crítico Universal, o Discursos varios en todo género de materias, para desengaño de errores comunes, Madrid, Real Compañía de Impresores y Libreros [disponible en ligne : http://filosofia.org/bjf].

—, 2014, Cartas eruditas y curiosas, I. Obras completas, t. II, éd. Inmaculada URZAINQUI et Eduardo SAN JosÉ VÁZQUEZ, Oviedo, Instituto Feijoo de Estudios del Siglo XVIII.

France Peter, 1992, Politeness and its Discontents: problems in French classical culture, Cambridge, Cambridge University Press.

FRANCO Gloria, 2005, « Bárbara de Braganza, la querella de las mujeres y la educación femenina », in M. Victoria LÓPEZ-CORDÓN \& Gloria FRANCO (dir.), La Reina Isabel y las reinas de España: realidad, modelos e imagen historiográfica, vol. 1, Madrid, Fundación Española de Historia Moderna, p. 497-522.

GOMIS Juan, 2009, «Feijoo y la literatura de cordel : crítica y exaltación de los errores comunes ", in Jesús AstigarRagA, María Victoria LÓPEZCORDÓN \& José María URKIA (dir.), Ilustración, vol. 3, Ilustraciones SaintSébastien, Real Sociedad Bascongada de Amigos del País, p. 321-340.

HAASE-Dubosc Danielle \& Marie-Élisabeth HENNEAU (dir.), 2013, Revisiter la querelle des femmes, vol. 2. Discours sur l'égalité/ inégalité des sexes, de 1600 à 1750, Saint-Ettienne, Publications de l'Université de Saint-Étienne. 
HAMERTON Katherine J., 2008, «Malebranche, Taste, and Sensibility: The Origins of Sensitive Taste and a Reconsideration of Cartesianism's Feminist Potential ", Journal of the History of Ideas, 69/4, p. 533-558.

—, 2010, «A feminist voice in the enlightenment salon: Madame de Lambert on taste, sensibility, and the feminine mind », Modern Intellectual History, 7/2, p. 209-238.

KITTS Sally Ann, 1995, The Debate on the Nature, Role and Influence of Woman in Eighteenth-century Spain, Lewiston, Edwin Mellen Press.

LA Vopa Anthony J., 2010, «Sexless Minds at Work and at Play: Poulain de la Barre and the Origins of Early Modern Feminism », Representations, $109 / 1$, p. $57-94$.

LOPEZ François, 2003, «Lecturas modernas y estructura de pensamiento », in Víctor INFANTES, François LOPEZ \& Jean-François BOTREL (dir.), Historia de la edición y de la lectura en España, 1472-1914, Madrid, Fundación Germán Sánchez Ruipérez, p. 445-446.

MACÍAS Marcelo (dir.), 1932, "Romance laudatorio dirigido por $\mathrm{D}^{\mathrm{a}}$ Ana María Moscoso de Prado al P. Feijoo, y carta de éste a la autora », Boletín de la Comisión Provincial de Monumentos Históricos y Artísticos de Orense, IX, 203, p. 324-328.

MACLEAN Ian, 1980, The Renaissance Notion of Woman: a study in the fortunes of scholasticism and medical science in European intellectual life, Cambridge, Cambridge University Press.

MARAÑÓN Gregorio, 1962 [1 $1^{\text {re }}$ éd. 1934], Las Ideas biologicas del Padre Feijoo, Madrid, Espasa Calpe.

MARTÍNEZ Martín, 1726, Carta defensiva que sobre el primer tomo del Teatro Crítico Universal ...le escribió su más aficionado amigo, Madrid, Imprenta Real.

MarTínez Vidal Álvar, 1987, «Los orígenes del mito de Oliva Sabuco en los albores de la Ilustración", Al-Basit : revista de estudios albacetenses, 22, p. 137-151.

Morvan De Bellegarde Jean-Baptiste, 1707, Lettres curieuses de littérature et morale, La Haye, Adrien Motjens.

ORTEGA Margarita, 1988, «La defensa de las mujeres en la sociedad del Antiguo Régimen: las aportaciones del pensamiento ilustrado ", in Pilar FOlguera (dir.), El feminismo en España: dos siglos de historia, Madrid, Fundación Pablo Iglesias, p. 3-28.

Pomata Gianna, 2010, «Introduction », in Oliva SABUCO, The True Medicine, éd. et trad. Gianna Pomata, Toronto, Toronto University Press, p. 1-86. 
—, 2013, "Was there a Querelle des Femmes in early modern medicine? ", Arenal. Revista de historia de las mujeres, 20/2, p. 313-341.

ROCHEFORT Florence \& Éliane VIENNOT (dir.), 2013, L'Engagement des hommes pour l'égalité des sexes (XIV -XXI siècle), Saint-Étienne, Publications de l'Université de Saint-Étienne.

SABUCO DE NANTES BARRERA Oliva, 1728, Nueva filosofía de la naturaleza del hombre, no conocida, ni alcanzada de los grandes filósofos antiguos..., Madrid, Imprenta Domingo Fernández.

SANTARELI Juan Antonio, [s.d.], Estrado Crítico en defensa de las mugeres contra el Theatro Crítico Universal de errores comunes [s.l., s.d.].

Smith Theresa Ann, 2006, The Emerging Female Citizen. Gender and Enlightenment in Spain, Berkeley, University of California Press.

StuUrman Siep, 2004, Francois Poullain de la Barre and the Invention of Modern Equality, Cambridge, MA, Harvard University Press.

—, 2005, «The deconstruction of gender: seventeenth-century feminism and modern equality », in Barbara TAYLOR \& Sarah KNOTT (dir.), Women, Gender and Enlightenment, London, Palgrave, p. 371-388.

TAusiet María, 2006, «De la ilusión al desencanto: Feijoo y los falsos posesos en la España del siglo XVIII », Historia Social, 54, p. 3-18.

URZAINQUI Inmaculada (dir.), 2003, Feijoo, boy. Semana Marañón 2000, Oviedo-Madrid, Instituto Feijoo de Estudios del Siglo XVIII-Fundación Gregorio Marañón.

—, 2014, "Estudio introductorio », in B.J. FEIJOO, Cartas eruditas y curiosas, I, Obras completas, t. II, ed. Inmaculada URZAINQUI \& Eduardo SAN JOSÉ VÁZQUEZ, Oviedo, Instituto Feijoo de Estudios del Siglo XVIII, p. 17-134.

VIENNOT Éliane (dir.), 2012, Revisiter la querelle des femmes. Discours sur l'égalité/ inégalité des femmes et des hommes, de 1750 aux lendemains de la Révolution française, Saint-Étienne, Publications de l'Université de Saint-Étienne.

ZAYAS Y SOTOMAYOR María, 2004, Novelas amorosas y ejemplares, éd. Julián Olivares, Madrid, Cátedra.

ZIMMERMANN Margarete, 2001, «The Querelle des Femmes as a cultural studies paradigm », in Anne Jacobson SCHUTTE, Thomas KUEHN \& Silvana SEIDEL MENCHI (dir.), Time, Space and Women's Lives in Early Modern Europe, Kirksville, Truman State University Press, p. 17-28. 